\title{
A Discrete-time Multivariable State Feedback MRAC Design with Application to Linearized Aircraft Models with Damage
}

\author{
Deepyaman Maiti, Jiaxing Guo and Gang Tao \\ Department of Electrical and Computer Engineering \\ University of Virginia \\ Charlottesville, VA 22904
}

\begin{abstract}
This paper studies the discrete-time multivariable model reference adaptive control (MRAC) design for discretetime linearized aircraft systems under damage conditions. A discrete-time sequential linear system with uncertain parameters and an unknown dynamics offset is used to model the large uncertain system parametric and structural changes caused by damage. The invariance properties of the two important design conditions, the interactor matrix and the signs of the high frequency gain matrix, of the discrete-time systems are studied. A discrete-time state feedback MRAC scheme is developed to ensure closed-loop signal boundedness and asymptotic output tracking in the presence of damage. Simulation study of the linearized NASA Generic Transport Model (GTM) shows the desired system performance under uncertain damage.
\end{abstract}

Keywords: Aircraft damage, digital adaptive control, discretization, flight control, GTM, linearization, output tracking, state feedback.

\section{INTRODUCTION}

In the research field of aircraft systems, study and control of aerodynamic and structural damage, such as locked flaps or loss of wing tips, are very pertinent topics. The designed controller must tolerate the damage, keep desired aircraft performance before and after damage, and guarantee aircraft safety. Considerable efforts have been devoted on this, e.g. ([1], [3], [4], [5], [6], [7], [8], [11]).

A damaged aircraft is a complicated nonlinear system with large uncertainties. We apply a linearization-based adaptive control design to deal with this. Due to uncertainties, equilibrium points are not available for the damaged system. We will arbitrarily choose an operating point to linearize the nominal and damaged systems. Thus the linearized system model with damage has an unknown constant dynamics offset. Digital control systems are widely used in flight control systems and they offer some advantages over conventional analog control, such as no degradation of performance because of wear and tear or aging. We will design a digital multivariable MRAC scheme for a linearized digital aircraft model with damage to compensate the uncertainties and make the output signals track given reference signals.

Discrete-time multivariable MRAC schemes have been well-studied ([9], [12]). For such designs, the two essential

Corresponding author: Gang Tao, gt9s@ virginia.edu.

This research was supported by NASA under the grant NNX08AB99A conditions are knowledge of the infinity zero structure of the system and that of signs of high frequency gain matrix principal minors. We will study these for a generic discrete-time aircraft model before and after damage and find that, irrespective of the relative degree of the elements of the interactor matrix of the continuous-time system, for sufficiently small sampling periods, the corresponding discretized system is unity. This means that even if the damage changes the continuous-time interactor matrix, the digital controller will tolerate the damage. Based on such system invariance, to compensate the unknown dynamics offset and the parametric uncertainties, we develop a discrete-time multivariable MRAC scheme with a state feedback controller to achieve asymptotic output tracking. This scheme has less restrictive matching conditions [2], so we can choose a common reference system before and after damage despite the uncertainties of damage. Stability analysis and simulation study of the linearized system obtained from the NASA GTM model will demonstrate that the proposed controller can guarantee boundedness of all closed-loop signals and asymptotical tracking of the reference by the output.

The paper is organized as follows. In Section II, we will give a discrete-time sequential linear system with an uncertain dynamics offset which represent the linearized aircraft system before and after damage. The invariance of the infinity zero structure is investigated in Section III. We will design an adaptive state feedback controller in Section IV, where a nominal controller is developed as a priori knowledge for the adaptive scheme. In Section V, we will apply the proposed scheme to a linearized GTM model with damage to demonstrate the desired tracking performance.

\section{Problem Statement}

We will consider a sequence of discrete-time linear systems with unknown dynamics offsets, which represent the linearized aircraft system models before and after damage.

\section{A. Control Problem}

Consider a sequential linear discrete-time system with $M$ inputs, $M$-outputs, $n$ states and an unknown dynamics offset

$$
x(k+1)=A x(k)+B u(k)+f_{0}, y(k)=C x(k),
$$

where $A, B, C$ are $n \times n, n \times M, M \times n$ unknown piecewise constant real matrices respectively with a finite number of 
unknown jumps $\left(A_{i}, B_{i}, C_{i}\right), i=1,2, \ldots, N$, and $f_{0}$, an $n$ dimensional real array, is an unknown piecewise constant offset with a finite number of unknown jumps $f_{0 i}, i=$ $1,2, \ldots, N$, such that $A=A_{i}, B=B_{i}, C=C_{i}, f_{0}=f_{0 i}$, for $k \in\left[k_{i-1}, k_{i}\right], i=1,2, \ldots, N . k_{0}=0$ and $k_{N}=\infty$.

Control objective. The control objective is to design a state feedback control law $u(k)$ in (1) such that all the closedloop signals remain bounded and the plant output signal $y(k)$ tracks a given reference output $y_{m}(k) \in R^{M}$ that is generated from the reference model system

$$
y_{m}(k)=W_{m}(z)[r](k) .
$$

$W_{m}(z)$ is an $M \times M$ real transfer matrix, and $r(k)$, an $M$ dimensional real array, is a bounded reference input signal.

Assumptions. To begin the control design, we assume:

(A1) All zeros of $G_{i}(z)=C_{i}\left(z I-A_{i}\right)^{-1} B_{i}, i=$ $1,2, \ldots, N$, lie within the unit circle in the $\mathrm{z}$-plane; (A2) $G_{i}(z), i=1,2, \ldots, N$, have full rank, there is a known modified left interactor matrix $\xi_{m}(z)$ for all $G_{i}(z), i=1,2, \ldots, N$, and the reference system transfer matrix $W_{m}(z)=\xi_{m}^{-1}(z)$; (A3) All leading principal minors $\Delta_{i j}, i=1,2, \ldots, N, j=1,2, \ldots, M$, of each high frequency gain matrices $K_{p i}=\lim _{z \rightarrow \infty} \xi_{m}(z) G_{i}(z)$ are nonzero and their signs are known, and $\operatorname{sign}\left[\Delta_{p j}\right]=$ $\operatorname{sign}\left[\Delta_{q j}\right], p, q=1,2, \ldots, N, j=1,2, \ldots, M$; (A4) $\left(A_{i}\right.$, $\left.B_{i}\right)$ is controllable and $\left(A_{i}, C_{i}\right)$ is observable.

Next, we need to show that the sequential linear digital system (1) can represent the linearized aircraft systems before and after damage, so that we can apply a linearization-based design for the control problem of nonlinear continuous-time aircraft systems with damage.

\section{B. Control of Nonlinear Aircraft Systems with Damage}

A nonlinear continuous-time aircraft system in nominal conditions can be described as

$$
\dot{x}(t)=f_{c 1}(x(t), u(t)), y(t)=C x(t)=[\theta, \psi]^{T},
$$

with state variables and input variables:

$$
x(t)=\left[u_{b}, w_{b}, q_{b}, \theta, v_{b}, r_{b}, p_{b}, \phi, \psi\right]^{T}, u(t)=\left[d_{e}, d_{r}\right]^{T},
$$

where $u_{b}, v_{b}$ and $w_{b}$ are the body-axis velocity components of the origin of the body-axis frame whose units are $\mathrm{ft} / \mathrm{sec}$, $p_{b}, q_{b}$ and $r_{b}$ are the body-axis components of the angular velocity in $\mathrm{rad} / \mathrm{sec}, \phi, \theta$ and $\psi$ are the Euler roll, pitch and yaw angles of the aircraft body axes with respect to the reference axes in radians, and $d_{e}$ and $d_{r}$ are the control surfaces' (elevator and rudder) angular positions in degrees.

Damage to the aircraft will cause uncertain parametric and structural variations. The nonlinear aircraft dynamic model in the presence of damage can be represented as

$$
\dot{x}(t)=f_{c 2}(x(t), u(t)), y(t)=C x(t)=[\theta, \psi]^{T},
$$

$f_{c 2}(t)$ is different from $f_{c 1}(t)$ in (3) due to the damage.

Linearized continuous-time system with damage. We choose $\left(x_{0}, u_{0}\right)$ as the operating point for linearizing the nonlinear aircraft system. The linearized nominal system at $\left(x_{0}, u_{0}\right)$ can be denoted as

$$
\Delta \dot{x}=A_{c 1} \Delta x+B_{c 1} \Delta u+f_{c 1}\left(x_{0}, u_{0}\right), \quad \Delta y=C \Delta x .
$$

The linearized damaged system at $\left(x_{0}, u_{0}\right)$ is expressed as

$$
\Delta \dot{x}=A_{c 2} \Delta x+B_{c 2} \Delta u+f_{c 2}\left(x_{0}, u_{0}\right), \quad \Delta y=C \Delta x,
$$

$\Delta x(t)=x(t)-x_{0}, \Delta y(t)=y(t)-C x_{0}, \Delta u(t)=u(t)-u_{0}$,

$\Delta x(t), \Delta y(t)$, and $\Delta u(t)$ being the linearized system's state, output, and input signals, and $x(t), y(t)$, and $u(t)$ are the nonlinear aircraft system's signals. Since $\left(x_{0}, u_{0}\right)$ can be chosen as the equilibrium point for the aircraft before damage, $f_{c 1}\left(x_{0}, u_{0}\right)=0$ in (6). $\left(x_{0}, u_{0}\right)$ is not the equilibrium point of the damaged system (5) and $f_{c 2}\left(x_{0}, u_{0}\right) \neq 0$. The parameters in the systems (6), (7) and the offset $f_{c 2}\left(x_{0}, u_{0}\right)$ are unknown due to the uncertainties in the aircraft system.

The linearized aircraft before and after damage can be respectively expressed as the two sequential linear systems:

$$
\begin{aligned}
\dot{x}(t) & =A_{c 1} x(t)+B_{c 1} u(t)+f_{c 1}, \quad y(t)=C x(t), \\
\dot{x}(t) & =A_{c 2} x(t)+B_{c 2} u(t)+f_{c 2}, \quad y(t)=C x(t),
\end{aligned}
$$

where $A_{c 1}, A_{c 2}, B_{c 1}, B_{c 2}, C$ are $n \times n, n \times n, n \times M, n \times M$, $M \times n$ unknown real matrices respectively. $f_{c 1}$ is a zero vector of dimension $n$, and $f_{c 2}$ is an n-dimensional real vector. The nominal system (6) or (8) is not coupled, so we consider the lateral-dimensional and longitudinal dynamics separately. But the damaged system (7) or (9) is coupled.

Discretization. The generic linearized aircraft systems (8) and (9) (before and after damage) both have the form

$$
\dot{x}(t)=A_{c} x(t)+B_{c} u(t)+f_{c}, \quad y(t)=C x(t),
$$

the subscript $c$ denoting continuous-time domain. The digital form of (10) can be derived as

$$
\begin{gathered}
x(k+1)=A x(k)+B u(k)+f_{0}, y(k)=C x(k), \\
A=I_{n}+T A_{c}+\frac{T^{2}}{2 !} A_{c}^{2}+\frac{T^{3}}{3 !} A_{c}^{3}+\cdots, \\
B=\left(T I_{n}+\frac{T^{2}}{2 !} A_{c}+\frac{T^{3}}{3 !} A_{c}^{2}+\frac{T^{4}}{4 !} A_{c}^{3}+\cdots\right) B_{c}, \\
f_{0}=\left(T I_{n}+\frac{T^{2}}{2 !} A_{c}+\frac{T^{3}}{3 !} A_{c}^{2}+\frac{T^{4}}{4 !} A_{c}^{3}+\cdots\right) f_{c} .
\end{gathered}
$$

The discrete digital systems before and after damage can be obtained. Thus, for the linearization-based design of the nonlinear aircraft system with damage, we consider the control problem of the system (1) in subsection II-A.

\section{INVARIANCE OF INFINITY ZERO STRUCTURES}

In this section, we examine the infinity zero structure invariance of the digital linearized system in presence of relatively small damages. 
Proposition 1. If, for an $M$-input, $M$-output continuoustime system, the $M \times M$ interactor matrix is diagonal and of the form

$$
\begin{aligned}
& \xi_{m}(s)=\operatorname{diag}\left\{(s+a)^{\rho_{1}},(s+a)^{\rho_{2}}, \ldots,(s+a)^{\rho_{M}}\right\}, \\
& \rho_{i}=1,2, \ldots, i=1,2, \ldots, M, a>0,
\end{aligned}
$$

then, the $M \times M$ interactor matrix of the corresponding $M$-input, $M$-output discretized system is of the form

$$
\xi_{m}(z)=\operatorname{diag}\left\{\left(z+a^{\prime}\right),\left(z+a^{\prime}\right), \ldots,\left(z+a^{\prime}\right)\right\},\left|a^{\prime}\right|<1 .
$$

for all small sampling intervals $T \in\left[0, T_{1}\right]$, with $T_{1}>0$.

Proof: Given the system matrices $A, B$ and $C$ of a linear continuous-time or discrete-time MIMO system with $n$ states, the transfer matrix can be calculated as

$$
\begin{aligned}
G(D) & =\frac{1}{\alpha(D)}\left(E_{n-1} D^{n-1}+E_{n-2} D^{n-2}+\cdots+E_{0}\right), \\
\alpha(D) & =\operatorname{det}(D I-A)=z^{n}+\alpha_{n-1} D^{n-1}+\cdots+\alpha_{0}, \\
E_{n-1} & =C B \\
E_{n-2} & =C A B+\alpha_{n-1} C B, \\
& \vdots \\
E_{0} & =C A^{n-1} B+\alpha_{n-1} C A^{n-2} B+\cdots+\alpha_{1} C B .
\end{aligned}
$$

For a continuous-time system, $D$ needs to replaced by $s$, and for a digital system, $D$ must be replaced by $z$. The elements of the interactor matrix $\xi_{m}(D)$ must be of relative degrees such that the high frequency gain matrix of $G(D)$, defined as $K_{p}=\lim _{D \rightarrow \infty} \xi_{m}(D) G(D)$ is finite and non-singular.

For a continuous-time system with system matrices $\left(A_{c}, B_{c}, C\right)$, the non-zero element in $i^{t h}$ row of the interactor matrix is $(s+a)^{\rho_{i}}$. This implies that

$$
E_{i, n-j}=C_{i} A_{c}^{j-1} B_{c}, j=1,2, \ldots, \rho_{i}-1
$$

are all zero vectors, and that

$$
E_{i, n-\rho_{i}}=C_{i} A_{c}^{\rho_{i}-1} B_{c},
$$

is not a zero vector. The subscript $i$ denotes the $i^{\text {th }}$ row of a matrix. Also, the high frequency gain matrix,

$$
K_{p_{c}}=\left[E_{1, n-\rho_{1}}, E_{2, n-\rho_{2}}, \ldots, E_{M, n-\rho_{M}}\right]^{T},
$$

is finite and non-singular, which means that the vectors $E_{1, n-\rho_{1}}, E_{2, n-\rho_{2}}, \ldots, E_{M, n-\rho_{M}}$ are linearly independent.

Now, for the discretized system,

$$
\begin{aligned}
E_{i,(n-1)} & =T C_{i} B_{c}+\frac{T^{2}}{2 !} C_{i} A_{c} B_{c}+\frac{T^{3}}{3 !} C_{i} A_{c}^{2} B_{c}+\cdots \\
& +\frac{T^{\rho_{i}}}{\rho_{i} !} C_{i} A_{c}^{\rho_{i}-1} B_{c}+\frac{T^{\rho_{i}+1}}{\left(\rho_{i}+1\right) !} C_{i} A_{c}^{\rho_{i}} B_{c}+\cdot(19)
\end{aligned}
$$

Considering (16) and (17), we note that the first $\rho_{i}-1$ terms on the right side of (19) are all zeros and contribute nothing. Thus, in the present case, for the discretized system,

$$
E_{i,(n-1)}=\frac{T^{\rho_{i}}}{\rho_{i} !} C_{i} A_{c}^{\rho_{i}-1} B_{c}+\frac{T^{\rho_{i}+1}}{\left(\rho_{i}+1\right) !} C_{i} A_{c}^{\rho_{i}} B_{c}+\cdots
$$

The first term on the right side of (20) is the same as the right side of (17) multiplied by the factor $\frac{T^{\rho_{i}}}{\rho_{i} !}$. Thus, the former is not a zero vector. We can conclude that there exists a $T_{1}>0$, such that, for all sampling periods $T \in\left[0, T_{1}\right]$, the effects of higher order terms (power of $T$ greater than $\rho_{i}$ ) can be nullified, that is $E_{i,(n-1)}$ of the discretized system is not a zero vector. Since the $E_{i,(n-1)}$ of the digital system is the $K_{i, p_{c}}$ in (18) multiplied by a scalar factor, the vectors $E_{i,(n-1)}, i=1,2, \ldots, M$ are linearly independent and $E_{n-1}$ of the digital system is non-singular.

For the discretized system, due to the non-singularity of $E_{n-1}$, each row of the transfer matrix $G(z)$ has a relative degree of 1 . So we choose the interactor matrix $\xi_{m}(z)$ as

$$
\xi_{m}(z)=\operatorname{diag}\left\{\left(z+a^{\prime}\right),\left(z+a^{\prime}\right), \ldots,\left(z+a^{\prime}\right)\right\},\left|a^{\prime}\right|<1,
$$

and the high frequency gain matrix is $K_{p_{d}}=E_{n-1} . \quad \nabla$

Proposition 2. The signs of the corresponding leading principal minors of the high frequency gain matrix remain unchanged after discretization for sufficiently small (as defined above) sampling intervals.

Proof: We noted that for sufficiently small sampling intervals, effects of higher order terms in $E_{n-1}$ (for the discretized system) can be neglected. Under such conditions, $K_{i, p_{d}} \approx$ $\frac{T^{\rho_{i}}}{\rho_{i} !} K_{i, p_{c}}, \rho_{i}$ being the relative degree of the diagonal element in the $i^{\text {th }}$ row of $\xi_{m}(s)$.

Lemma 1: The signs of the determinants of corresponding $j \times j$ sub-matrices of $K_{p_{c}}$ and $K_{p_{d}}$ are the same, when any $j$ (corresponding) elements from each of $j$ (corresponding) row of $K_{p_{c}}$ or $K_{p_{d}}$ form the said sub-matrices, with $j=$ $1,2, \ldots, M$.

The logic behind the lemma statement can be grasped if we keep in mind the one-to-one correspondence between the elements of $K_{p_{c}}$ and $K_{p_{d}}$. The lemma can be proved using mathematical induction.

Proposition 2 being a special case of lemma 1, we see that signs of corresponding leading principal minors of the high frequency gain matrix are unaffected by discretizaton. $\quad \nabla$

Propostions 1 and 2 imply that the assumptions (A2) and (A3) hold for the aircraft system (1), for which we will derive a multivariable MRAC scheme next.

\section{Adaptive Control Scheme}

In this section, we will present a multivariable state feedback model reference adaptive control design for the linear sequential discrete-time system (1).

State feedback controller design. To compensate the constant offset term $f$ in (1), we choose the state feedback controller structure as

$$
u(k)=K_{1}^{T}(k) x(k)+K_{2}(k) r(k)+k_{3}(k),
$$

where $k_{3}(k) \in R^{M}$ is the adaptive estimate of an unknown constant compensation term $k_{3}^{*}$, which will be derived next, for canceling the effect of the constant offset $f$, and $K_{1}(k)$ and $K_{2}(k)$ are the estimates of the nominal $K_{1}^{*}$ and $K_{2}^{*}$ which satisfy the matching conditions

$$
C\left(z I-A-B K_{1}^{* T}\right)^{-1} B K_{2}^{*}=W_{m}(z), K_{2}^{*-1}=K_{p},
$$


where $K_{p}$ is the piecewise constant high frequency gain matrix, for each jump, $K_{p}=K_{p i}=\lim _{z \rightarrow \infty} \xi_{m}(z) G_{i}(z), i=$ $1,2, \ldots, N$.

Remark 1: From assumption (A2), all $\left(A_{i}, B_{i}, C_{i}\right), i=$ $1,2, \ldots, N$, have the same interactor matrix $\xi_{m}(z)$, and $W_{m}(z)=\xi_{m}^{-1}(z)$. Based on [10], for each $\left(A_{i}, B_{i}, C_{i}\right), i=$ $1,2, \ldots, N$, we can obtain a set of constant parameters $K_{1}^{*}$ and $K_{2}^{*}$ to satisfy the plant-model matching equations (22), so that the nominal $K_{1}^{*}$ and $K_{2}^{*}$ are piecewise constants.

To derive $k_{3}^{*}$, we apply a nominal controller

$$
u(k)=K_{1}^{* T} x(k)+K_{2}^{*} r(k)+k_{3}^{*}
$$

to the system (1) to achieve exact plant-model matching.

To define the matching parameter vector $k_{3}^{*}$, we consider a particular set of constant values of the system parameters $(A, B, f)$. Then, substituting (23) in the plant (1), we have the closed-loop system in the $z$-domain as

$$
\begin{aligned}
y(z) & =C\left(z I-A-B K_{1}^{* T}\right)^{-1} B K_{2}^{*} r(z)+\Delta(z), \\
\Delta(z) & =C\left(z I-A-B K_{1}^{* T}\right)^{-1}\left(B \frac{k_{3}^{*} z}{z-1}+\frac{f_{0} z}{z-1}\right) .
\end{aligned}
$$

From the reference system (2) and the matching conditions (22), we have the output tracking error in the $z$-domain as

$$
e(z)=y(z)-y_{m}(z)=\Delta(z) .
$$

Applying the $z$-domain final value theorem, we obtain

$$
\lim _{k \rightarrow \infty} e(k)=\lim _{z \rightarrow 1}(z-1) \Delta(z)=D k_{3}^{*}+d
$$

for some constant invertible matrix $D$ and vector $d$. For offset rejection, we set

$$
k_{3}^{*}=-D^{-1} d,
$$

and then from (27)-(28), we have

$$
\lim _{k \rightarrow \infty}\left(y(k)-y_{m}(k)\right)=\lim _{k \rightarrow \infty} \delta(k)=0
$$

exponentially fast, where $\delta(k)=\mathcal{Z}^{-1}[\Delta(z)]$.

Since the system parameters $\left(A, B, f_{0}\right)$ are piecewise constant, the nominal matching parameter $k_{3}^{*}$ is also piecewise constant, as defined above for each set of $\left(A, B, f_{0}\right)$.

From the matching conditions (22) and (28), we can conclude that there exists a nominal controller (23) to achieve the asymptotic output tracking. However, we will use the adaptively updated control law (21), since the parameters $K_{1}^{*}, K_{2}^{*}$, and $k_{3}^{*}$ are unknown. In the following, we will first develop the tracking error equation by applying the control law (21) to the system (1).

Tracking error equation. Substituting the control law (21) in the plant (1), we have

$$
\begin{aligned}
x(k+1)= & \left(A+B K_{1}^{* T}\right) x(k)+B K_{2}^{*} r(k)+B k_{3}^{*}+f_{0} \\
& +B\left(\widetilde{K}_{1}^{T}(k) x(k)+\widetilde{K}_{2}(k) r(k)+\tilde{k}_{3}(k)\right) \\
y(k)= & C x(k),
\end{aligned}
$$

where $\widetilde{K}_{1}^{T}(k)=K_{1}^{T}(k)-K_{1}^{*}, \widetilde{K}_{2}(k)=K_{2}(k)-K_{2}^{*}$, and $\tilde{k}_{3}(k)=k_{3}(k)-k_{3}^{*}$.
In view of the reference model (2), matching conditions (22), (28), and (30), the output tracking error is

$$
e(k)=y(k)-y_{m}(k)=W_{m}(k) K_{p}\left[\widetilde{\Theta}^{T} \omega\right](k)+\delta(k),
$$

where

$$
\begin{aligned}
\widetilde{\Theta}(k) & =\Theta(k)-\Theta^{*}, \quad \Theta(k)=\left[K_{1}^{T}(k), K_{2}(k), k_{3}(k)\right]^{T}, \\
\Theta^{*} & =\left[K_{1}^{* T}, K_{2}^{*}, k_{3}^{*}\right]^{T}, \omega(k)=\left[x^{T}(k), r^{T}(k), 1\right]^{T} .
\end{aligned}
$$

To deal with the uncertainty of the high frequency gain matrix $K_{p}$, we use its LDS decomposition [12]. A matrix $K_{p} \in R^{M \times M}$ with all its leading principal minors being non-zero has a unique decomposition:

$$
K_{p}=L_{s} D_{s} S
$$

Remark 2: In the adaptive laws design, we will use $D_{s}$ matrix as a gain matrix. Although $K_{p}$ is a piecewise constant, we can choose a uniform $D_{s}$ for all the high frequency gain matrices from the assumption (A3) for the adaptive laws.

Substituting the LDS decompensation of $K_{p}$ (with a uniform $D_{s}$ ) (33) in (31), and ignoring the exponentially decaying term $\delta(t)$, we have

$$
L_{s}^{-1} \xi_{m}(z)[e](k)=D_{s} S \tilde{\Theta}^{T}(k) \omega(k) .
$$

We introduce a filter $h(z)=1 / f_{h}(z)$, where $f_{h}(z)$ is a stable and monic polynomial of degree equals to the degree of $\xi_{m}(z)$, Operating both sides of (34) by $h(z) I_{M}$ leads to

$$
L_{s}^{-1} \xi_{m}(z) h(z)[e](k)=D_{s} S h(z)\left[\tilde{\Theta}^{T} \omega\right](k) .
$$

To parameterize the unknown matrix $L_{s}$, we introduce

$$
\Theta_{0}^{*}=L_{s}^{-1}-I=\theta_{i j}^{*},
$$

where $\theta_{i j}^{*}=0$ for $i=1,2, \ldots, M$ and $j \geq i$. Then we have

$$
\begin{aligned}
& \bar{e}(k)+\left[0, \theta_{2}^{* T} \eta_{2}(k), \theta_{3}^{* T} \eta_{3}(k), \ldots, \theta_{M}^{* T} \eta_{M}(k)\right]^{T} \\
= & D_{s} S h(z)\left[\tilde{\Theta}^{T} \omega\right](k),
\end{aligned}
$$

where

$$
\begin{aligned}
\bar{e}(k) & =\xi_{m}(z) h(z)[e](k)=\left[\bar{e}_{1}(k), \ldots, \bar{e}_{M}(k)\right]^{T}, \\
\eta_{i}(k) & =\left[\bar{e}_{1}(k), \ldots, \bar{e}_{i-1}(k)\right]^{T} \in R^{i-1}, i=2, \ldots, M \\
\theta_{i}^{*} & =\left[\theta_{i 1}^{*}, \ldots, \theta_{i i-1}^{*}\right]^{T}, i=2, \ldots, M
\end{aligned}
$$

Estimation error. From (37), we introduce the estimation error signal

$$
\begin{aligned}
\epsilon(k)= & {\left[0, \theta_{2}^{T}(k) \eta_{2}(k), \theta_{3}^{T}(k) \eta_{3}(k), \ldots, \theta_{M}^{T}(k) \eta_{M}(k)\right]^{T} } \\
& +\Psi(k) \xi(k)+\bar{e}(k),
\end{aligned}
$$

where $\theta_{i}(k), i=2,3, \ldots, M$ are the estimates of $\theta_{i}^{*}$, and $\Psi(k)$ is the estimate of $\Psi^{*}=D_{s} S$, and

$$
\xi(k)=\Theta^{T}(k) \zeta(k)-h(z)\left[\Theta^{T} \omega\right](k), \zeta(k)=h(z)[\omega](k) .
$$

From (37)-(42), we can derive that

$$
\begin{aligned}
\epsilon(k)= & {\left[0, \tilde{\theta}_{2}^{T}(k) \eta_{2}(k), \tilde{\theta}_{3}^{T}(k) \eta_{3}(k), \ldots, \tilde{\theta}_{M}^{T}(k) \eta_{M}(k)\right]^{T} } \\
& +D_{s} S \tilde{\Theta}^{T}(k) \zeta(k)+\tilde{\Psi}(k) \xi(k),
\end{aligned}
$$


where $\tilde{\theta}_{i}(k)=\theta_{i}(k)-\theta_{i}^{*}, i=2,3, \ldots, M$, and $\tilde{\Psi}(k)=$ $\Psi(k)-\Psi^{*}$ are the related parameter errors.

Adaptive laws. With the estimation error model (43), we choose the adaptive laws

$$
\begin{aligned}
\theta_{i}(k+1) & =\theta_{i}(k)-\frac{\Gamma_{\theta i} \epsilon_{i}(k) \eta_{i}(k)}{m^{2}(k)}, i=2,3, \ldots, M(44) \\
\Theta^{T}(k+1) & =\Theta^{T}(k)-\frac{D_{s} \epsilon(k) \zeta^{T}(k)}{m^{2}(k)}, \\
\Psi(k+1) & =\Psi(k)-\frac{\Gamma \epsilon(k) \xi^{T}(k)}{m^{2}(k)},
\end{aligned}
$$

where the signal $\epsilon(k)=\left[\epsilon_{1}(k), \epsilon_{2}(k), \ldots, \epsilon_{M}(k)\right]^{T}$ is computed from (41), $0<\Gamma_{\theta i}=\Gamma_{\theta i}^{T}<2 I_{i-1}\left(\Gamma_{\theta i} \in\right.$ $\left.R^{(i-1) \times(i-1)}\right), i=2,3, \ldots, M$, and $0<\Gamma=\Gamma^{T}<2 I_{M}$, and $D_{s}$ is defined in (31) with $\gamma_{i}>0, i=2,3, \ldots, M$, satisfying the condition $0<D_{s} U^{T} D_{s}^{i-1} D^{*} U D_{s}<I_{M}$ are adaptation gain matrices. The normalization signal is

$m(k)=\left(1+\zeta^{T}(k) \zeta(k)+\xi^{T}(k) \xi(k)+\sum_{i=2}^{M} \eta_{i}^{T}(k) \eta_{i}(k)\right)^{1 / 2}$

Stability analysis. From the adaptive laws (44)-(46), we have the following desired stability properties.

Lemma 2: The adaptive laws (44)-(46) ensure that

(i) $\theta_{i}(k) \in L^{\infty}, i=2,3, \ldots, M, \Theta(k) \in L^{\infty}, \Psi(k) \in$ $L^{\infty}$, and $\frac{\epsilon(k)}{m(k)} \in L^{2} \cap L^{\infty}$;

(ii) $\theta_{i}\left(k+i_{0}\right)-\theta_{i}(k) \in L^{2} \cap L^{\infty}, i=2,3, \ldots, M, \Theta(k+$ $\left.i_{0}\right)-\Theta(k) \in L^{2} \cap L^{\infty}$, and $\Psi\left(k+i_{0}\right)-\Psi(k) \in L^{2} \cap L^{\infty}$, for any finite integer $i_{0}>0$.

Proof: Consider a positive definite function

$$
V=\sum_{i=2}^{M} \tilde{\theta}_{i}^{T} \Gamma_{\theta i}^{-1} \tilde{\theta}_{i}+\operatorname{tr}\left[\tilde{\Psi}^{T} \Gamma^{-1} \tilde{\Psi}\right]+\operatorname{tr}\left[\tilde{\Theta} S \tilde{\Theta}^{T}\right],
$$

which has a finite jump at $k_{i}, i=1,2, \ldots, N-1$, i.e.,

$$
V\left(k_{i}+1\right)-V\left(k_{i}\right)<\infty .
$$

For

$$
M_{\eta}(k)=\operatorname{diag}\left\{0, \eta_{1}^{T}(k) \Gamma_{\theta 1} \eta_{1}(k), \ldots, \eta_{M}^{T}(k) \Gamma_{\theta M} \eta_{M}(k)\right\},
$$

the time-increment of $V$ in $\left[k_{i-1}, k_{i}\right], i=1,2, \ldots, N$, is

$$
\begin{aligned}
& V\left(\tilde{\theta}_{i}(k+1), \tilde{\Psi}(k+1)\right), \tilde{\Theta}(k+1)-V\left(\tilde{\theta}_{i}(k), \tilde{\Psi}(k), \tilde{\Theta}(k)\right) \\
& \leq-\alpha_{1} \frac{\epsilon^{T}(k) \epsilon(k)}{m^{2}(k)}
\end{aligned}
$$

for some constant $\alpha_{1}<0$. That is $V\left(k_{i}\right) \leq V\left(k_{i-1}\right)$, and from (49), we can conclude that $V(k)$ is bounded for $[0, \infty]$. The last inequality in (51) is due to the conditions that $0<$ $\Gamma_{\theta i}=\Gamma_{\theta i}^{T}<2 I_{i-1}, i=2,3, \ldots, M, 0<\Gamma=\Gamma^{T}<2 I_{M}$, and $0<D_{s} S D_{s}=D_{s} U^{T} D_{s}^{-1} D^{*} U D_{s}<2 I_{M}$, and the choice of $m(k)$ in (47).

Therefore, from (51), $\theta_{i}(k) \in L^{\infty}, i=2,3, \ldots, M$, $\Theta(k) \in L^{\infty}, \Psi(k) \in L^{\infty}, \frac{\epsilon(k)}{m(k)} \in L^{2} \cap L^{\infty}$ (using (43)).
From the adaptive laws (44)-(46) and the results of lemma 2(i), $\theta_{i}(k+1)-\theta_{i}(k) \in L^{2} \cap L^{\infty}, i=2,3, \ldots, M, \Theta(k+$ 1) $-\Theta(k) \in L^{2} \cap L^{\infty}$, and $\Psi(k+1)-\Psi(k) \in L^{2} \cap L^{\infty}$. Finally, use of the inequality

$$
\left\|\chi\left(k+i_{0}\right)-\chi(k)\right\|_{2} \leq \sum_{i=0}^{i_{0}-1}\|\chi(k+i+1)-\chi(k+i)\|_{2}
$$

allows us to prove the second part of lemma 2 .

These properties allow us to prove the following theorem.

Theorem 1: The multivariable MRAC scheme with the state feedback control law (21) updated by the adaptive laws (44) (46), when applied to the plant (1), guarantees the closedloop signal boundedness and asymptotic output tracking: $\lim _{k \rightarrow \infty}\left(y(k)-y_{m}(k)\right)=0$, for any initial conditions.

This is proved using a small gain theory [12]. A key step in the procedure is to express a filtered version of the output $y(k)$ in a feedback framework which has a small gain due to the $L^{2}$ properties of $\Theta\left(k+i_{0}\right)-\Theta(k), \theta_{i}\left(k+i_{0}\right)-\theta_{i}(k)$ and $\frac{\epsilon(k)}{m(k)}$. The state feedback control $u(k)$ depends on the states $x(k)$. So we must express it in terms of the output $y(k)$ (and the input $u(k)$ itself through a dynamic block). This is done using a state observer representation of the plant: $x(k+1)=$ $(A-L C) x(k)+B u(k)+L y(k)+f_{0}$ for a gain matrix $L \in$ $R^{n \times M}$ such that $A-L C$ is stable (which is possible because $(A, C)$ is observable). Then, the analysis procedure in [12] is used to conclude the closed-loop signal boundedness and asymptotic output tracking: $\lim _{k \rightarrow \infty}\left(y(k)-y_{m}(k)\right)=0$ for the state feedback case.

\section{Aircraft Flight Control Application}

In this section, we will apply the above MRAC scheme to the linearized NASA generic transport model (GTM) under nominal and damaged conditions. The GTM model contains several damage scenarios; in this study, we choose the damage case with loss of outboard left wing-tip.

System description. We linearize the nominal and the damaged GTM models at an operating point $\left(x_{0}, u_{0}\right)$, where $x_{0}=\left[u_{b 0}, w_{b 0}, 0, \theta_{0}, 0,0,0,0, \psi_{0}\right]^{T}$, and $u_{0}=\left[d_{e 0}, d_{r 0}\right]^{T}$. To obtain such an operating point, the nominal GTM is trimmed at a wings-level steady-state flight condition with the equivalent airspeed as 90 knots and roll angle as 0 radian.

The nominal and damaged linearized aircraft systems are of the forms (8) and (9) respectively. We discretize the continuous-time systems presented in [3] with sampling interval $T=0.001$ seconds to obtain the discrete-time nominal and damaged aircraft systems

$$
\begin{array}{ll}
x(k+1)=A_{1} x(k)+B_{1} u(k)+f_{01}, & y(k)=C x(k) . \\
x(k+1)=A_{2} x(k)+B_{2} u(k)+f_{02}, & y(k)=C x(k) .
\end{array}
$$

The exact expressions for $A_{1}, B_{1}, C, A_{2}, B_{2}, f_{02}$ are omitted owing to paucity of space.

Verification of design conditions. In Section III, the invariance properties are obtained based on generic models. In the simulation studies, we will further verify the design conditions using numerical values. All zeros of $G_{1}(z)=$ 
$C\left(z I-A_{1}\right)^{-1} B_{1}$ and $G_{2}(z)=C\left(z I-A_{2}\right)^{-1} B_{2}$ are inside the unit circle, and $G_{1}(z)$ and $G_{2}(z)$ have full rank.

We can choose a common interactor matrix for both $G_{1}(z)$ and $G_{2}(z)$ as $\xi_{m}(z)=\operatorname{diag}\{(z-0.5),(z-0.5)\}$, such that the high frequency gain matrix for the nominal case is

$$
K_{p 1}=\lim _{z \rightarrow \infty} \xi_{m}(z) G_{1}(z)=10^{-6} \times\left[\begin{array}{cc}
-0.5421 & 0 \\
0 & -0.2980
\end{array}\right]
$$

and the high frequency gain matrix for the damaged case is

$$
K_{p 2}=\lim _{z \rightarrow \infty} \xi_{m}(z) G_{2}(z)=10^{-4} \times\left[\begin{array}{cc}
-0.5264 & -0.0076 \\
0.0153 & -0.3043
\end{array}\right] .
$$

Signs of first leading principal minor of $K_{p 1}$ and $K_{p 2}$ (before and after damage) are $\operatorname{sign}\left(\Delta_{11}\right)=\operatorname{sign}\left(\Delta_{21}\right)=-1$, and signs of second leading principal minor are $\operatorname{sign}\left(\Delta_{21}\right)=$ $\operatorname{sign}\left(\Delta_{22}\right)=1$. This verifies the sign invariance.

Reference model. From the common interactor matrix $\xi_{m}(z)$ for both nominal and damage cases, we choose the transfer matrix of the reference model (2) as $W_{m}(z)=$ $\xi_{m}^{-1}(z)=\operatorname{diag}\{1 /(z-0.5), 1 /(z-0.5)\}$.

Design parameters. Since the degree of $\xi_{m}(z)$ is 1 , we choose the filter $h(z)=1 / z$. For the adaptive laws (44)(46), we choose $\Gamma_{\theta 2}=1.5, \Gamma=\operatorname{diag}\{1.5,1.5\}$, and $D_{s}=$ $\operatorname{diag}\{-0.75,-1\}$ due to sign invariance of principal minors.

Simulation results. The reference input is $r(k)=$ $[8 \pi / 9,15 \pi / 18]^{T}$. Applying the control law (21) with the adaptive laws (44)-(46), we check that (Fig. 1) the output $y(k)=[\theta(k), \psi(k)]^{T}$ (solid) tracks the reference $y_{m}(k)=$ $\left[\theta_{m}(k), \psi_{m}(k)\right]^{T}$ (dotted) after damage at 300 seconds.

We verify the controller for varying reference inputs as well. With the reference input $r(k)=$ $[2.5 \sin (0.015 k T), 2.5 \sin (0.015 k T)]^{T}$, we see that (Fig. 2) the output tracks the reference after damage at 450 seconds.

The transient responses and the initial oscillations depend on the gain parameters chosen.

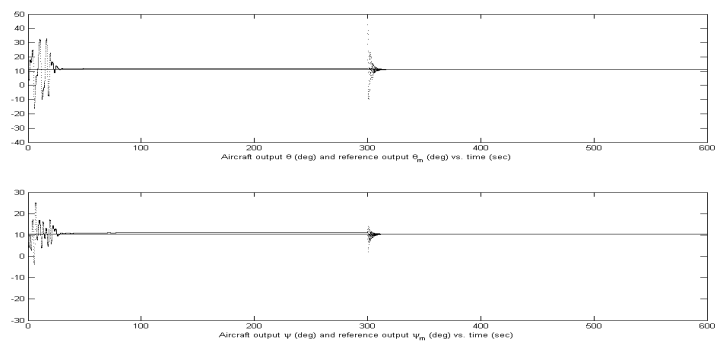

Fig. 1. Aircraft outputs (solid) vs. reference outputs (dotted) (Case I).

\section{Vi. Conclusions}

In this paper, we developed a discrete-time multivariable state feedback for output tracking MRAC scheme for a linearized aircraft model with damage. We linearized and discretized both nominal and damaged aircraft systems at a given operating point to obtain a discrete-time sequential linear system with a dynamics offset. Study of the infinity
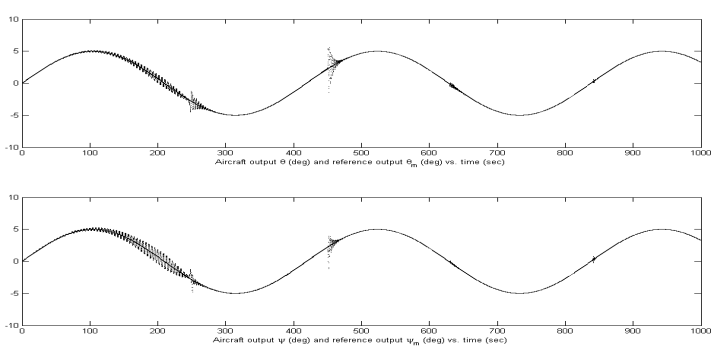

Fig. 2. Aircraft outputs (solid) vs. reference outputs (dotted) (Case II).

zero structure of the discretized system before and after damage ensured that the invariance conditions for multivariable MRAC design were met. We designed an adaptive state feedback digital controller to compensate the parametric uncertainties and the offset, and make the signals of closedloop system bounded and the output signals track some reference signals. A simulation study of the linearized system obtained from the GTM model verified desired performance.

\section{REFERENCES}

[1] Bacon, B. J. and I. M. Gregory, "General equations of motion for a damaged asymmetric aircraft," Proc. of AIAA Guidance, Navigation, and Control Conference, No. AIAA-2007-6306, 2007.

[2] Guo, J. X., G. Tao, and Y. Liu, "A multivariable MRAC scheme with application to a nonlinear aircraft model," Automatica, vol. 47, Issue 4, pp. 804-812, April 2011.

[3] Guo, J. X, Y. Liu, and G. Tao, "A Multivariable MRAC design using state feedback for linearized aircraft models with damage," Proceedings of 2010 American Control Conference, pp. 2095-2100, Baltimore, MD, June 30-July 22010.

[4] Liu, Y. and G. Tao, "Model-based direct adaptive actuator failure compensation techniques with applications to aircraft flight control systems," Proc. of AIAA Guidance, Navigation, and Control Conference, No. AIAA-2006-6554, 2006.

[5] Liu, Y. and G. Tao, "Multivariable MRAC for aircraft with abrupt damages," Proceedings of 2008 American Control Conference, June 2008, pp. 2981-2986.

[6] Liu, Y. and Tao, G., "Multivariable MRAC using Nussbaum gains for aircraft with abrupt damages," Proceedings of 47th IEEE Conference on Decision and Control, 2008.

[7] Liu, Y. and G. Tao and S. M. Joshi, "Modeling and model reference adaptive control of aircraft with asymmetric damages," Proceedings of 2009 AIAA Guidance, Navigation and Control Conference, Paper AIAA-2009-5617, Chicago, IL, August 2009.

[8] Lombaerts, T. J. J., Chu, Q. P., Mulder, J. A., and Joosten, D. A., "Real time damaged aircraft model identification for reconfiguring flight control," Proc. of AIAA Atmospheric Flight Mechanics Conference, No. AIAA-2007-6717, 2007.

[9] Lu, Y., Tao, G., and Abdelzaher, T., "LDU Parametrized Discrete Time Multivariable MRAC and Application to a Web Cache System," IEEE Conference on Decision and Control, Las Vegas, Nevada, December 2002.

[10] Morse, A. S., "Parametrizations for Multivariable Adaptive Control," Proc. 20th IEEE CDC, pp. 970-972, San Diego, CA, 1981.

[11] Nguyen, N., Krishnakumar, K., Kaneshige, J., and Nespeca, P., "Dynamics and adaptive control for stability recovery of damaged asymmetric aircraft," Proceedings of AIAA Guidance, Navigation, and Control Conference, No. AIAA-2006-6049, 2006.

[12] Tao, G., Adaptive Control Design and Analysis, John Wiley and Sons, New York, 2003. 